\title{
www.ijpsonline.com \\ Safety and Clinical Efficacy of Hepatectomy for Left Hepatolithiasis
}

\author{
Q. ZOU, J. CHEN AND C. LI* \\ Department of Hepatobiliary Surgery, Shanghai Pudong Hospital, Fudan University Pudong Medical Center, 2800 Gongwei \\ Road, Huinan Town, Pudong, Shanghai 201399, China
}

Zou et al.: Safety and effectiveness of Hepatectomy for Left Hepatolithiasis

\begin{abstract}
The present study was aimed to explore the safety and clinical efficacy of hepatectomy for left hepatolithiasis. A total of 200 patients with hepatolithiasis were selected as the research subjects to analyze the characteristics of hepatolithiasis. The research results showed that most patients with hepatolithiasis were suffering from regional lithiasis in the left half of the liver and the excellent rate of postoperative therapeutic effects of hepatectomy reached $92.0 \%$. In addition, it was found through the subsequent postoperative follow-ups that among all the hepatolithiasis patients who underwent hepatectomy, 5 died of the recurrence of lithiasis or the associated causes, with the mortality rate being $2.5 \%$, and no patients died during the operation processes. Therefore, based on these results, it could be concluded that hepatectomy is a safe and effective treatment for hepatolithiasis with lower risk and better prognosis.
\end{abstract}

Key words: Hepatolithiasis, excision surgeries, typing

Hepatolithiasis refers to the primary hepatolithiasis in which stones are originated in the intrahepatic bile ducts and excludes the lithiasis where stones are originated in the gallbladder and moved up to the intrahepatic bile duct, as well as the hepatolithiasis caused by cholestasis and inflammation of biliary tract secondary to bile duct injury-originated bile duct stenosis, biliary dilatation and bile duct deformations ${ }^{[1]}$. Hepatolithiasis is a common disease in China, especially in the vast areas of South China, Southwest China, Yangtze River Basin and Southeast Coast. Due to its complicated lesions, high stone residual rate, high recurrence rate and high re-operation rate, hepatolithiasis often causes serious complications and becomes an important cause of death of benign biliary tract diseases in China; its treatments are mainly surgical in nature with the principle of to remove the lesions and the stones, to correct the stenosis, to smoothen the drainage and to prevent the recurrence ${ }^{[2,3]}$. The application of comprehensive treatment has significantly improved the overall clinical efficacy of hepatolithiasis; in addition, the residual and recurrence rate of stones has been significantly reduced and the quality of life of patients has been greatly improved. However, in general, hepatectomy is still the major treatment of hepatolithiasis. The surgical methods for hepatolithiasis mainly include 4 different approaches of stone removal, the bile duct incision, the partial hepatectomy, the repair and rebuilding of bile duct stenosis and liver transplantation. In recent years, hepatectomy has been widely applied as the treatment of hepatolithiasis.

The important clinicopathological feature of hepatolithiasis is the tree-like distribution of stones along with the intrahepatic lesions of the bile duct. The predilection sites of intrahepatic bile duct lithiasis are the left outer lobe, the right anterior lobe and the right posterior lobe, most of which are accompanied by stenosis or dilation of intrahepatic bile ducts, or the liver fibrotic atrophy, the empyema of biliary ducts and carcinogenesis ${ }^{[4]}$. Therefore, early hepatolithiasis is mostly regional. In recent years, studies have shown that hepatectomy is the treatment that follows the above principle the best; it has obvious advantages compared to other non-hepatectomy treatments and can completely remove the lesions including stones ${ }^{[5,6]}$. In addition, due to the stimulations of stones, cholestasis and bacterial infection are important pathogenic factors for cholangiocarcinoma, therefore, hepatectomy can also effectively prevent the stonerelated cholangiocarcinoma. Recently, the concept of being minimally invasive is also reflected in the treatment of regional hepatolithiasis ${ }^{[7,8]}$. Various hospitals have achieved good therapeutic effects in the treatment of intrahepatic bile duct stones with laparoscopic hepatectomy, which has the advantages of 
being minimally invasive leading to less postoperative pain, less intraoperative bleeding, quick recovery and shorter term of hospital stay. However, laparoscopic hepatectomy has certain limitations. At present, its indications are for intrahepatic bile duct stones, intrahepatic lesions, fibrotic atrophy, or although there is no obvious liver surface fibrotic atrophy, but combined with bile duct stricture, stones and relatively limited lesions located in the lower left or right lobe, especially the left lobe is most suitable.

A total of 200 patients with hepatolithiasis treated with left hepatectomy were investigated. The safety and effectiveness of the treatment was explored, with an intention to provide evidence for this form of treatment of hepatolithiasis. Two hundred patients with hepatolithiasis who were admitted to Shanghai Pudong Hospital from September 2017 to January 2019 were included in this study. The characteristics of extrahepatic resection of various types of hepatolithiasis were analyzed and the treatment plans were summarized. The informed consent signed by the patients or their family members was obtained and this study was approved by the medical ethics committee of Shanghai Pudong Hospital.

The inclusion criteria were, based the Guidelines for Diagnosis and Treatment of Hepatolithiasis established by the Chinese Medical Association in 2007, hepatolithiasis refers to the primary lithiasis in which stones are originated in the intrahepatic bile ducts, as well as the hepatolithiasis caused by cholestasis and the inflammation of biliary tract, which are the results of the secondary bile duct diseases of injury-originated bile duct stenosis, biliary dilatation, and bile duct deformations, and are diagnosed as hepatolithiasis after postoperative pathological analysis; the commonly applied operation is hepatectomy, including various regular and irregular hepatectomy, which could be combined with biliary incision and stone removal, biliary stenosis repair and reconstruction, and intrabiliary drainage; it could include the combined hepatic cysts, hemangioma, hepatic abscess, Caroli's disease, and cases of hepatic cholangiocarcinoma that were not diagnosed before surgery but were diagnosed by intraoperative or postoperative pathological analysis. The exclusion criteria were as follows: Hepatic cholangiocarcinoma was diagnosed before surgery; the main lesion of the resected hepatic lobe was not intrahepatic bile duct stones but hepatic cysts, hemangioma, Caroli's disease, and other lesions.
The treatments for hepatolithiasis are mainly surgeries and operations, which include the bile duct incision, the partial hepatectomy, the repair and rebuild of bile duct stenosis, and liver transplantation. The typing of hepatolithiasis itself is an important basis for determining the surgical approach. The hepatolithiasis is classified into 3 major types, the static type, the obstruction type, and the cholangitis type; hepatolithiasis patients of the static type often have no obvious symptoms or mild symptoms, with pain or discomfort only in the upper abdomen; the static hepatolithiasis is often found in the physical examination. In terms of the obstruction type, the manifestations include intermittent jaundice, continuous pain and discomfort in the areas of liver, chest and abdomen, digestive dysfunction, and other symptoms of biliary obstruction; in addition, the bilateral hepatolithiasis with hepatic bile duct stenosis may present with persistent jaundice. In terms of the cholangitis type, the manifestations include recurrent and acute purulent cholangitis; an acute attack of cholangitis hepatolithiasis is manifested as paroxysmal cramps or persistent pain, chills, fever, jaundice; right upper quadrant tenderness, hepatic septal pain, hepatomegaly, and tenderness. The severe cases may be associated with sepsis manifestations; the number of neutrophilic granulocytes and leukocytes in peripheral blood would increase, serum transaminase sharply increases, and the serum bilirubin, alkaline phosphatase and glutamyl transpeptidase would also increase. No jaundice or slight jaundice could occur in case of onesided hepatic duct lithiasis combined with acute hepatic cholangitis and the serum bilirubin could be at a normal level or slightly elevated; no symptoms nor obstructive manifestations are seen during the intermittent period.

Hepatolithiasis was typed into, type I or the regional type, in which stones are distributed in one or several hepatic segments along the intrahepatic bile duct tree, often with stenosis of the hepatic duct and atrophy of the affected liver segment; the clinical manifestations included the static type, the obstruction type, or the cholangitis type, as shown in fig. 1A and B. Type II or the diffuse type was characterized by stones distributed all over the bilateral hepatic bile duct, as shown in fig. 1C; based on the lesions of liver parenchyma, it is further divided into 3 subtypes, type IIa, the diffuse type without significant liver fibrosis and atrophy. Type IIb, the diffuse type with regional liver parenchymal fibrosis and atrophy, usually associated with atrophy of the main hepatic duct of the atrophic liver segment. Type IIc, the diffuse type with extensive fibrosis of liver 
parenchyma to form secondary biliary cirrhosis and portal hypertension, usually accompanied by severe stenosis of the left and right hepatic ducts or the bile duct below the confluence. The type $\mathrm{E}$, the additive type in which combined extrahepatic bile duct lithiasis can be found as shown in fig. 1D; based on the functional state of the Oddi sphincter, it is further divided into 3 subtypes, type Ea, where Oddi sphincter is normal; type $\mathrm{Eb}$, where Oddi sphincter is in relaxation and type Ec, where Oddi sphincter is narrowed.

Based on the distribution of intrahepatic stones, the additive hepatolithiasis is further divided into the type III, which included type IIIa, type IIIb and type IIIc, and the treatments of intrahepatic lesions are the same as the non-type E cases; therefore, these are classified into type IVa and type IVb, respectively. The benign stenosis of the bile duct is classified according to Matsumoto typing, as shown in fig. 2.

Patients were submitted to type-B ultrasonography and computed tomography (CT). The type-B ultrasonography is the primary imaging examination approach of hepatolithiasis; CT scans could comprehensively show the distribution of intrahepatic bile duct stones, bile duct system expansion, and liver parenchyma lesions and can also obtain the stereotactic conformation of the intrahepatic bile duct system as well as the 3D distribution of intrahepatic stones.

Nutritional support was given to patients before surgery to improve their nutritional status and the reserve liver

A
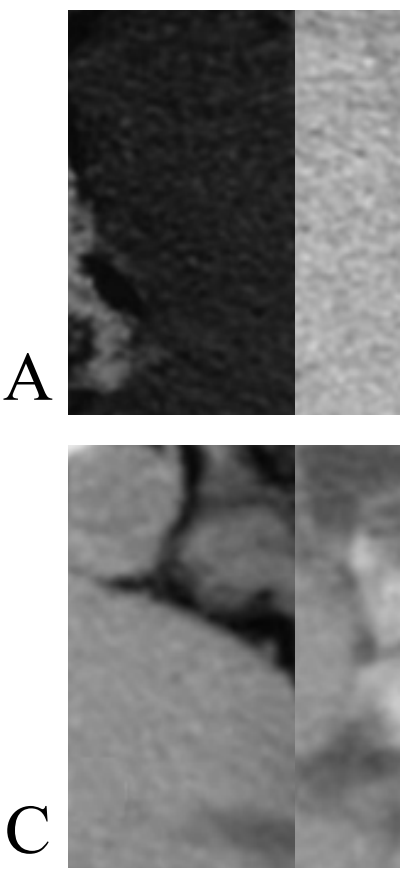

Fig. 1: Hepatolithiasis types

A, B. regional hepatolithiasis, $C$. diffuse hepatolithiasis and D. additive hepatolithiasis
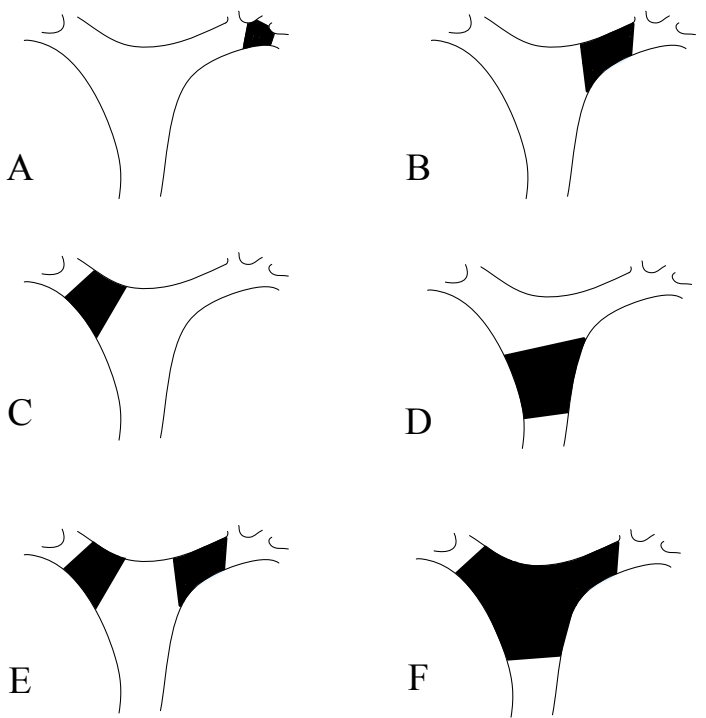

Fig. 2: Benign stenosis of bile duct

A. Type I with left outer lobe bile duct opening, B. type II, left hepatic duct orifice, C. type III right hepatic anterior lobe or upper anterior lobe, D. type IVa the upper segment of the common hepatic duct, E. type IVb left and right hepatic ducts, F. type IVc mainly located in the upper part of the common hepatic duct, combined with left and right hepatic stenosis

function; in addition, broad-spectrum antiinfective treatment was given to patients with biliary tract infection. The patients were treated with general anesthesia through the combined inhalation method; based on the intraoperative percussion, preoperative type-B ultrasonography, CT, the typing, location, and degree of the stones were further determined; the bile duct was fully exposed, the stones were removed; in terms of patients whose porta hepatis were deep, the quadrate lobe was removed to fully expose the bile duct and the stones were removed; in terms of patients whose hepatic stones were difficult to be totally removed, or patients with the liver parenchymal fibrotic atrophy or the liver tissues were associated with caroli, hemangioma, hepatic abscess, hepatic cholangiocarcinoma, the corresponding tissues were removed through hepatectomy. All cases were given antiinfective treatment according to bile bacterial culture and drug sensitivity test after the surgeries.

The SPSS 13.0 statistics software package was applied to organize and descriptively analyze the data. The measurement data were expressed as mean \pm standard deviation, the ordered multi-group independent samples were tested by the rank sum, $\mathrm{p}<0.05$ indicated obviously statistical significance.

A total of 200 cases were analyzed, in which male patients accounted for $32.5 \%$ (65) and female patients accounted for $67.5 \%$ (135); the average age was 53.6, with the maximum of 80 and the minimum of 28 . In 
addition, the hepatic function stratification indicated 148 cases of level A, 52 cases of level B, and 0 cases of level C. Hospital stay ranged from 12 to $75 \mathrm{~d}$, with an average of $23.2 \mathrm{~d}$. The composition of different typing cases was shown in fig. 3. The type I hepatolithiasis cases were 116, the type IIa hepatolithiasis cases were 28 , the type IIb hepatolithiasis cases were 47, the type IIc hepatolithiasis cases were 9 and the composition of different case types of bile duct stenosis was shown in fig. 4.

Of all the bile duct stenosis cases, the left hepatic duct orifice stenosis cases (type II) were the most common.

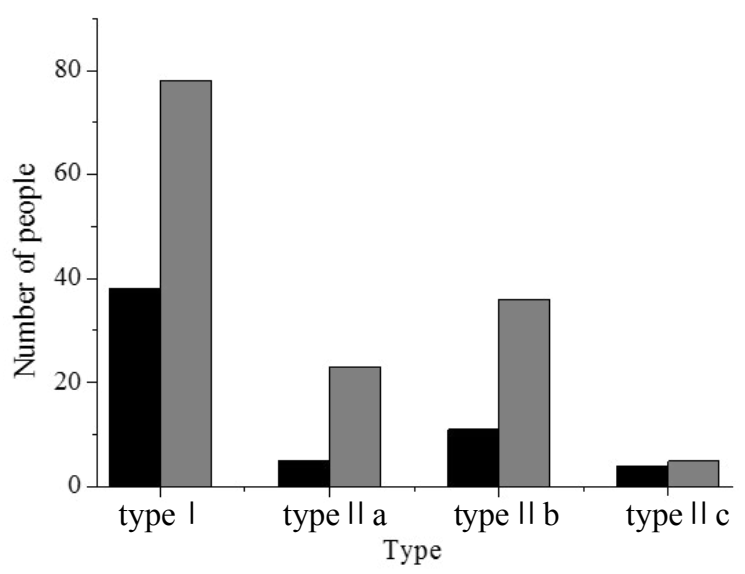

Fig. 3: Distribution of patients with different case types (匹) Extrahepatic biliary stone and ( $\square$ ) type $E$
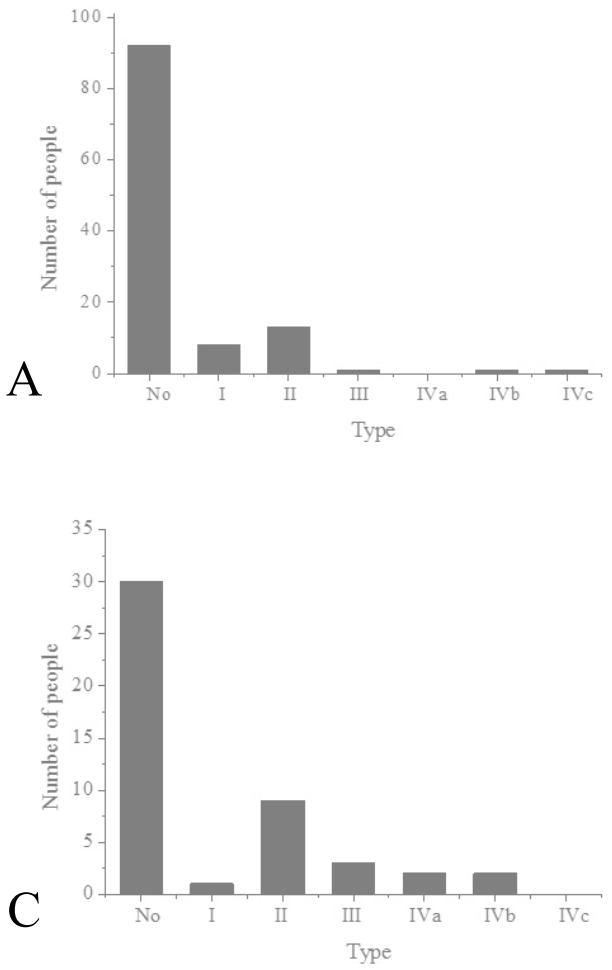

Clinical manifestations were categorized as follows, of all the cases in the group, the static cases were 5; the obstruction cases were 92; the cholangitis cases were 103, the detailed distribution was shown in fig. 5A. The preoperative stratification of hepatic function was shown in fig. 5B, and other preoperative lesions of the liver were shown in fig. $5 \mathrm{C}$.

Postoperative pathological analysis showed that the left hepatolithiasis was the most common type and the bilateral intrahepatic bile duct stones were mostly found in the left liver. Different types of hepatolithiasis should be treated with different approaches of hepatectomy and the proportion of left hepatectomy was the largest. All cases presented intrahepatic bile duct pigment stones, bile duct fibrous tissue and small bile duct hyperplasia, fibrous bile duct wall hyperplasia and chronic inflammatory changes. Four patients had different degrees of cholestasis and sclerotic changes in the liver, 1 patient developed hepatocyte steatosis and 9 patients developed cholangiocarcinoma.

Postoperative complications, management and outcomes indicated that 36 patients had postoperative complications, in which 13 patients had postoperative incision infection that was gradually healed through dressing changes of the wounds and antiinfective treatment. In addition, 11 cases of liver infection were
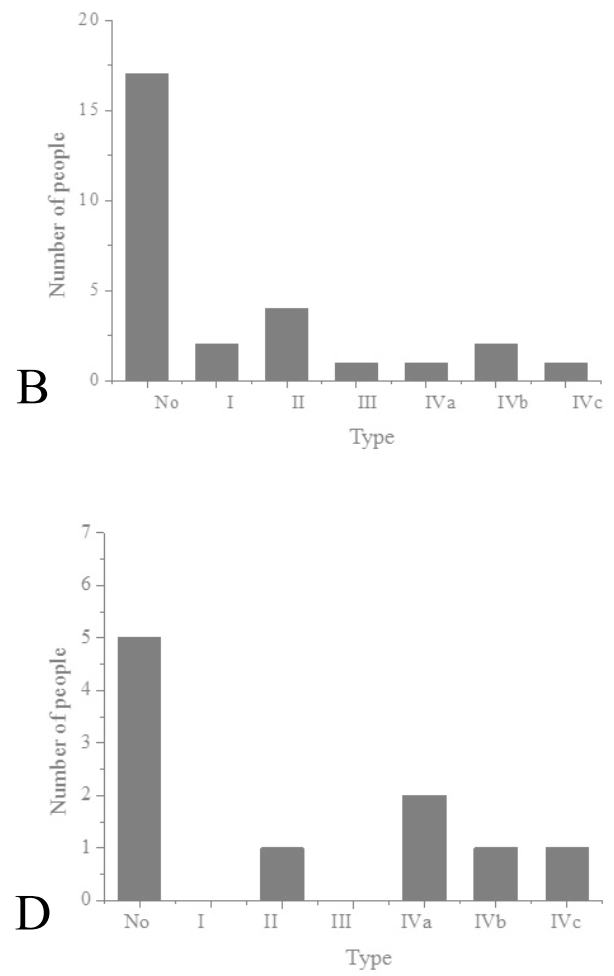

Fig. 4: Composition of different types of bile duct stenosis

A. Composition of type I bile duct stenosis of hepatolithiasis, B. composition of type IIA bile duct stenosis of hepatolithiasis, C. composition of type IIB bile duct stenosis of hepatolithiasis and D. composition of type IIC bile duct stenosis of hepatolithiasis 

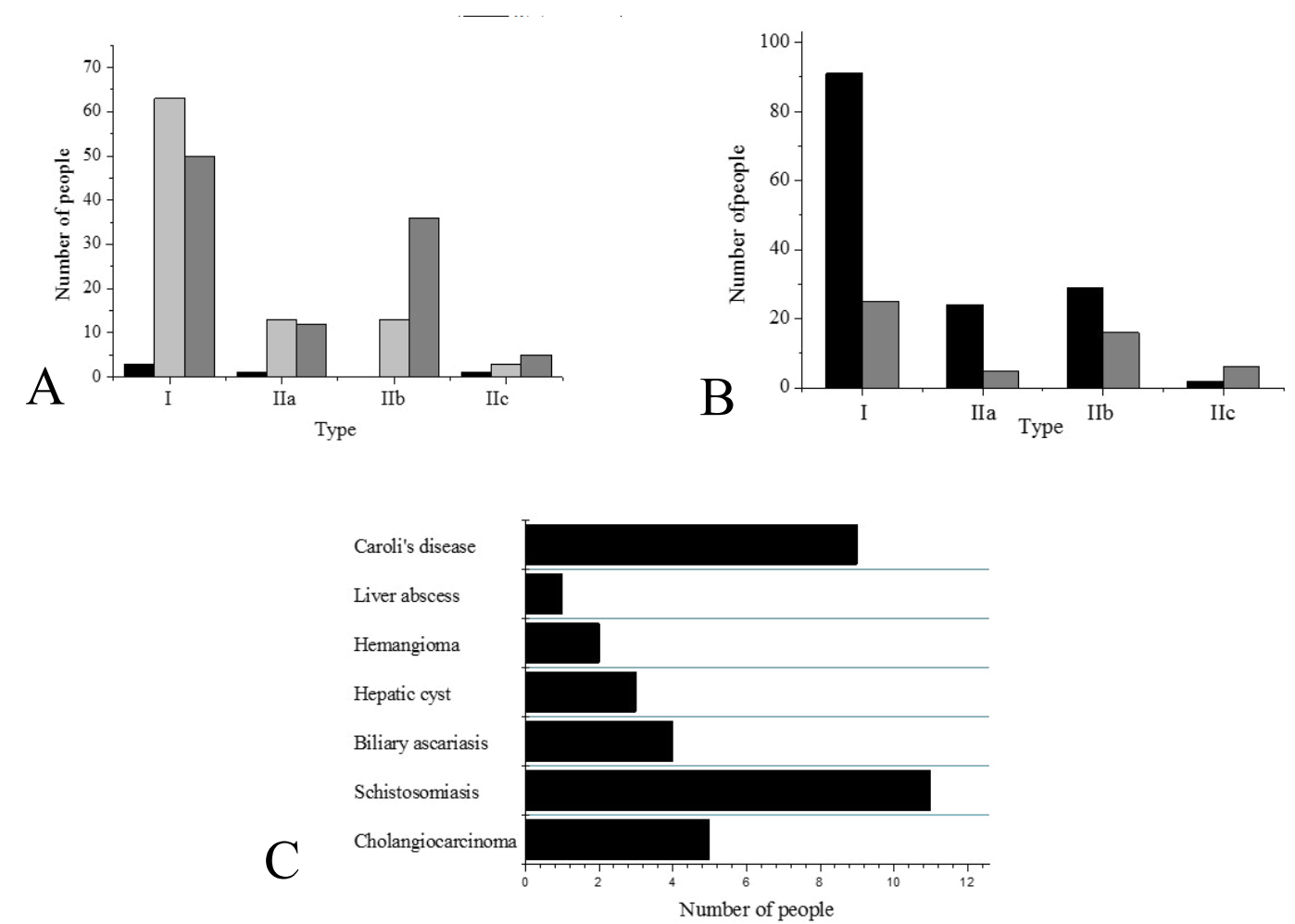

Fig. 5: Classification of hepatic bile duct stenosis and other liver lesions A. Clinical manifestation typing, ( $\square)$ stationary, $(\square)$ obstructive, ( $\square$ ) cholangitis; B. preoperative hepatic function stratification, ( $\square$ ) level A, ( $\square)$ level B and C. other preoperative lesions of the liver

cured through drainage, wound dressing changes and antiinfective treatment. Further, 4 cases of bile leakage and 1 case of pancreatic leakage occurred. Moreover, 5 patients developed pulmonary infection, which was through atomization and antiinfective drugs based on the sputum bacterial culture, 1 patient developed stress ulcer, which was healed after acidification, hemostasis and fluid replacement and 1 patient died of multiple organ failure due to biliary sepsis.

After T-tube angiography or ultrasonography, it was found that 31 out of 200 patients $(15.5 \%)$ had residual stones, which were located in the left liver in 13 cases, in the right liver in 9 cases, and in both the left and right liver in 9 cases; all the residual stones were located in the third grade bile duct and above.

Postoperative follow-ups were done through telephone, the clinical efficacy and mortality of 200 patients were shown in fig. 6. The total good and excellent outcome incidence was $92 \%$. The overall analysis indicated that hepatectomy was an effective method for the treatment of hepatolithiasis, compared to the clinical efficacy of other treatment methods $(\mathrm{p}<0.05)$.

The total excellent and good outcome fully reflected that the hepatectomy is an effective method for the treatment of hepatolithiasis; the reason is that hepatectomy fully conforms to the treatment principle to remove the lesions and the stones, to correct the stenosis, to smooth the drainage, and to prevent the recurrence. The removal of lesions is the most important principle since it is based on the thorough comprehension of the pathophysiology of hepatolithiasis.

Mortality of patients observed in the follow up was divided into death due to the disease and death due to other causes; of all the deaths caused by the disease, 1 patient died of postoperative biliary sepsis, 2 patients died of the hepatic functional failure caused by the biliary cirrhosis, which was induced by the repeated recurrence of cholangitis due to the recurrence of lithiasis; in the other 6 cases, 3 patients died of the complicated cholangiocarcinoma in the late stage, 2 patients died of other systematic diseases such as colon cancer and 1 patient died from other causes such as a car accident.

Of all the 200 hepatolithiasis patients analyzed in this study, female patients were a majority at $67.5 \%$ and the average age of incidence was $53.6 \mathrm{y}$. It was found that the hepatolithiasis was mostly the type I (regional hepatolithiasis), while the type II and type E were relatively fewer. Hepatolithiasis is a common disease of bile duct system with complicated lesions; in addition, its residual stone rate, recurrence rate, and re-operation rate are high, leading to serious complications. 


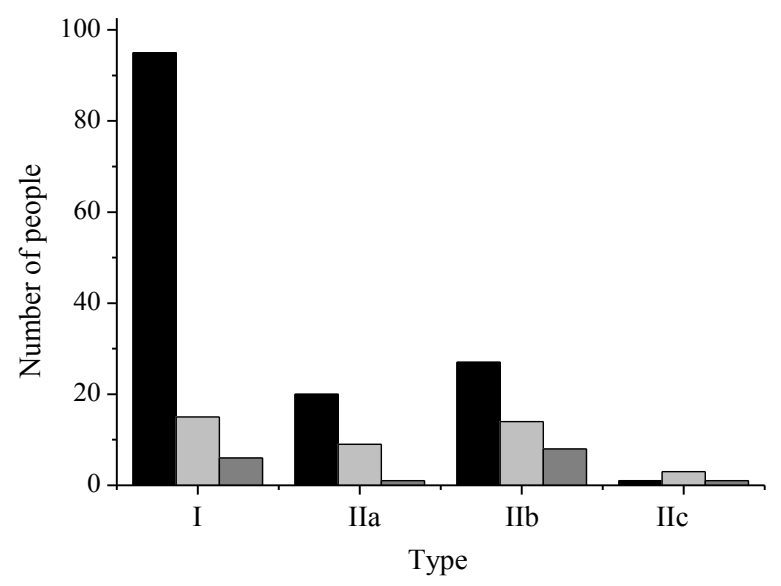

Fig. 6: Clinical efficacy of different types of hepatolithiasis (a) Excellent, ( $\square$ ) good, ( $\square$ ) bad

Recently, hepatectomy has been widely practiced to treat hepatolithiasis. The results of this investigation showed that left hepatolithiasis is the largest component of hepatolithiasis and left hepatectomy is the most common treatment. Successful treatment outcome after hepatectomy was found to be $92.0 \%$ (184/200), which is mainly applied to the most predilection sites of hepatolithiasis. Through the postoperative followups of 200 patients, it was found that only 5 patients died of hepatolithiasis and 6 patients died due to other causes; thus, the postoperative mortality was only $2.5 \%$. Therefore, it is proved that left hepatectomy is safe and effective treatment with a high incidence of positive therapeutic outcome for left hepatolithiasis.

\section{Acknowledgements:}

This work was supported by Funded by Shanghai Municipal Commission of Health and Family Planning (Grant No.201640384).

\section{REFERENCES}

1. Jegadeesan M, Goyal N, Gupta S. Middle Hepatic Vein Bleed During Donor Hepatectomy: Technique for Safe Practice. J Clin Exp Hepatol 2017;7(4): S0973688316305199.

2. Langiewicz M, Schlegel A, Saponara E. Hedgehog pathway mediates early acceleration of liver regeneration induced by a novel two-staged hepatectomy in mice. J Hepatol 2017;66(3): 560-570

3. Kim JH, Choi JW. A Modified Liver Hanging Maneuver in Pure Laparoscopic Left Hemihepatectomy with Preservation of the Middle Hepatic Vein: Video and Technique. J Gastrointest Surg 2017; 21(7): 1-5.

4. Matsushita K, Gotoh K, Eguchi H. Agenesis of the left hepatic lobe undergoing laparoscopic hepatectomy for hepatocellular carcinoma: a case report. Surg Case Rep 2017;3(1): 50.

5. Haldrup D, Heebøll S, Thomsen KL. Preserved liver regeneration capacity after partial hepatectomy in rats with non-alcoholic steatohepatitis. World J Hepatol 2018;10(1):821.

6. Li Q, Zhao Q, Zhang C. The ileal FGF15/19 to hepatic FGFR4 axis regulates liver regeneration after partial hepatectomy in mice. J Phys Biochem 2018;(1):1-14.

7. Abramovitch R, Nachmansson N, Frede S. SAT-412 - Impaired liver regeneration following hepatectomy and bleeding is associated with a shift from hepatocyte proliferation to hypertrophy. J Hepatol 2017; 66(1): S615.

8. Sikalias $\mathrm{N}$, Karatzas $\mathrm{T}$, Alexiou K. Intermittent Ischemic Preconditioning Protects Against Hepatic IschemiaReperfusion Injury and Extensive Hepatectomy in Steatotic Rat Liver. J Investig Surg 2017;(2):1-12.

This is an open access article distributed under the terms of the Creative Commons Attribution-NonCommercial-ShareAlike 3.0 License, which allows others to remix, tweak, and build upon the work non-commercially, as long as the author is credited and the new creations are licensed under the identical terms

This article was originally published in a special issue: Special issue on "Drug Development and Human Health in China"

Indian J Pharm Sci 2020:82(1)spl issue2;92-97 